\title{
Giant Pulmonary Bulla Underlying Bronchopulmonary Dysplasia in a Very Low-birth- weight Infant: a Case Report
}

Tamao Shinohara ( $\sim$ shinotamao@hotmail.co.jp)

Yamanashi Prefecture Central Hospital: Yamanashi Kenritsu Chuo Byoin https://orcid.org/0000-0003-

0714-1239

\section{Yohei Hasebe}

University of Yamanashi Hospital: Yamanashi Daigaku Igakubu Fuzoku Byoin

Daisuke Watanabe

Yamanashi Prefecture Central Hospital: Yamanashi Kenritsu Chuo Byoin

\section{Fuminori Numano}

Yamanashi Prefecture Central Hospital: Yamanashi Kenritsu Chuo Byoin

\section{Tomohiro Saito}

Yamanashi Prefecture Central Hospital: Yamanashi Kenritsu Chuo Byoin

Keiichi Koizumi

Yamanashi Prefecture Central Hospital: Yamanashi Kenritsu Chuo Byoin

Atsushi Nemoto

Yamanashi Prefecture Central Hospital: Yamanashi Kenritsu Chuo Byoin

Tomoko Tandou

Yamanashi Prefecture Central Hospital: Yamanashi Kenritsu Chuo Byoin

Toshio Oyama

Yamanashi Prefecture Central Hospital: Yamanashi Kenritsu Chuo Byoin Noboru Oyachi

Yamanashi Prefecture Central Hospital: Yamanashi Kenritsu Chuo Byoin

Minako Hoshiai

Yamanashi Prefecture Central Hospital: Yamanashi Kenritsu Chuo Byoin

\section{Atsushi Naito}

Yamanashi Prefecture Central Hospital: Yamanashi Kenritsu Chuo Byoin

\section{Case report}

Keywords: Acquired cystic lung disease, Bronchopulmonary dysplasia, Giant pulmonary bulla, Infant, Pulmonary interstitial emphysema 
Posted Date: April 1st, 2021

DOI: https://doi.org/10.21203/rs.3.rs-378547/v1

License: (c) (i) This work is licensed under a Creative Commons Attribution 4.0 International License. Read Full License 


\section{Case report}

2

3 Giant pulmonary bulla underlying bronchopulmonary dysplasia in a very low-birth-weight

4 infant: A case report

5

6 Tamao Shinohara, M.D., Ph.D. ${ }^{1)}$; Yohei Hasebe, M.D., Ph.D. ${ }^{2)}$; Daisuke Watanabe, M.D. ${ }^{1)}$;

7 Fuminori Numano, M.D. ${ }^{3)}$; Tomohiro Saito, M.D.,Ph.D. ${ }^{4)}$; Keiichi Koizumi, M.D.,

8 Ph.D. ${ }^{3)}$; Atsushi Nemoto, M.D., Ph.D. 1); Tomoko Tandou, M.D., Ph.D. ${ }^{4) ;}$ Toshio

9 Oyama, M.D., Ph.D. ${ }^{5)}$; Noboru Oyachi, M.D., Ph.D. ${ }^{3)}$; Minako Hoshiai, M.D., Ph.D. ${ }^{4)}$;

10 Atsushi Naito M.D., Ph.D. ${ }^{1)}$

11

12 1) Department of Neonatology, Yamanashi Prefectural Central Hospital, Yamanashi, Japan

13 2) Department of Pediatrics, Graduate School of Medicine, University of Yamanashi,

14 Yamanashi, Japan

15 3) Department of Pediatric Surgery, Yamanashi Prefectural Central Hospital, Yamanashi, 16 Japan

17 4) Department of Pediatrics, Yamanashi Prefectural Central Hospital, Yamanashi, Japan

18 5) Department of Pathology, Yamanashi Prefectural Central Hospital, Yamanashi, Japan

19

20

21 Short running title: Giant pulmonary bulla in infant

22

23 Abstract word count: 252 words

24 Main text word count (background through discussion): 1130 words

\section{Number of text pages:}


26 Total number of tables and figures: 3 figures, 0 tables

27

28 *Correspondence to: Tamao Shinohara, M.D., Ph.D.

29 Department of Neonatology, Yamanashi Prefectural Central Hospital, Yamanashi, 1-1-1

30 Fujimi, Kofu, Yamanashi 400-8506, Japan

$31 \quad$ Tel.: $+8155-253-7111$

32 Fax: +81 55-253-8031

33 E-mail: t-shinohara1181@yamanashi.pref.jp

34

35

36

37

38

39

40

41

42

43

44

45

46

47

48 
50 Abstract

51 Background: Acquired cystic lung disease is a serious respiratory complication of

52

53

54

55

56

57 bullectomy.

58 disease in infants. 
73 Keywords: Acquired cystic lung disease, Bronchopulmonary dysplasia, Giant pulmonary

74 bulla, Infant, Pulmonary interstitial emphysema

75

76

77

78

79

80

81

82

83

84

85

86

87

88

89

90

91

92

93

94

95

\section{Background}

Acquired cystic lung disease in premature infants is a serious respiratory complication of bronchopulmonary dysplasia (BPD) [1]. This includes various pathologies such as pulmonary interstitial emphysema (PIE) [2-4], pulmonary bulla [5], and pneumatocele [6]. Among acquired cystic lung diseases, most cases are reported as PIE. However, bullous lung diseases are rare in infants. Since the treatment strategies for these pathologic conditions are different, it is important to establish a correct diagnosis. Here, we report a rare case of giant pulmonary bulla in a very low-birth-weight infant and highlight the utility of computed tomography $(\mathrm{CT})$ in deciding the therapeutic strategy.

\section{Case presentation}

A male infant $(525 \mathrm{~g})$ with no congenital pulmonary cysts detected on foetal echography was born at 23 weeks of gestation with low Apgar scores of 1, 3, and 6 at 1, 5, and $10 \mathrm{~min}$, respectively. After birth, the patient was intubated and received synchronised intermittent mandatory ventilation. Initial chest radiographs at birth showed ground-glass appearance and air bronchograms with no cystic lesion consistent with respiratory distress syndrome. The patient was then was treated with surfactant. On the $11^{\text {th }}$ day, he developed pneumothorax on the right side, which required the placement of a chest drain. On the $24^{\text {th }}$ day, the lung cyst appeared and gradually expanded in the right upper lobe. Because of the recurrences of pneumothorax, the mechanical ventilator mode was changed to neurally adjusted ventilatory assist or high-frequency oscillatory ventilation to reduce ventilator- 
97 induced lung injury. On the $76^{\text {th }}$ day, he was extubated and continued to receive non-invasive respiratory support as a consequence of BPD. However, the lung cysts gradually expanded. Chest CT performed on the fifth month of age revealed two types of cystic lesions. The first was a huge solitary cyst with an empty interior and smooth outlines below the pleura. The second was multicystic lesions of various sizes with septations located adjacent to the first. Due to mass effects, there was a left mediastinal shift with left lung atelectasis (Fig. 1). These cysts were diagnosed as giant pulmonary bulla with PIE. In the interim, his respiratory condition remained stable with oxygen therapy and his body weight gradually increased. He was then discharged from the hospital at 6 months of age. After that, we observed his clinical condition while planning to perform surgical intervention should his respiratory condition deteriorate.

At 9 months of age, he was admitted due to acute bronchitis caused by human metapneumovirus. A chest radiograph showed further expansion of the giant pulmonary bulla triggered by the respiratory infection (Fig. 2). His clinical condition could not be controlled by medical treatment such that he presented with respiratory failure and obstructive shock. While PIE is expected to spontaneously regress, the giant pulmonary bulla was thought to be the main cause of respiratory failure. This warranted cystectomy without resection of the PIE. The giant pulmonary bulla was incised, and a few air leakage sites were sutured directly. The cyst sac was empty without remnants of the lung tissues. The visceral pleura was then reinforced using a residual cyst wall. Histopathologic examination of the excised bulla wall revealed that it was derived from the fibrous tissue and was located within the subpleural lung parenchyma. The giant emphysematous cyst was histologically diagnosed as a bulla, excluding congenital cystic lung disease (Fig. 3).

After the operation, the patient had no respiratory problems and did not need any respiratory support. He is currently three years old and is doing well. 
123 Discussion and conclusions

Bulla is defined radiologically as an emphysematous space with a diameter of $1 \mathrm{~cm}$

or more and is morphologically located in the subpleural lung parenchyma [7]. Reid et al.

proposed a classification for bulla. Type I bulla is characterised pathologically as a narrow-

necked empty sac with a clear linear outline in the absence of vascular or airway remnants [7].

The outer surface of the bulla is composed of visceral pleura, while its inner surface is

composed of fibrous tissue, derived from the pleura and the destroyed pulmonary tissue [8].

In our case, the expanded cyst was a monocyst with a clear rim of the wall on CT. The cystic

wall histologically contained fibrous tissue and was located within the subpleural lung

132 parenchyma. The cyst was diagnosed as a Reid type I bulla based on both radiologic and

133 histopathologic findings. The symptomatic expanded cyst met the following criteria of a giant

134 pulmonary bulla: (1) upper lobe involvement, (2) occupying at least one-third of the

135 hemithorax, and (3) compressing the surrounding lung parenchyma [9]. On the contrary,

136 multiple cystic spaces were adjacent to the giant pulmonary bulla. These showed extra-

137 alveolar air accumulation and a line-and-dot pattern, which is a specific sign of PIE on CT.

138 The mechanism of PIE is likely from air leakage into the perivascular and

139 peribronchial spaces due to the high airway pressure of mechanical ventilation [10]. This PIE

140 may spread centrifugally along the bronchovascular sheath or lymphatic channels. Air leakage

141 develops into expanded airspaces of the terminal bronchiole and alveolar septal destruction,

142 which leads to subpleural emphysema [10]. Therefore, the pathogenesis of bulla and the

143 adjacent PIE, in this case, was considered to be closely related.

144 In the management of acquired cystic lung disease, PIE may be reversible.

145 Conservative treatment by selective intubation, selective bronchial obstruction, or decubitus

146 positioning is accepted as the initial management [3-5]. However, the giant pulmonary bulla 
147 can be considered for surgery $[2,8,11]$. Based on the good outcome of our case, cystectomy

148 was considered to be safe and effective as it can leave sufficient residual lung in small infants.

149 In our case, the chest radiograph could not sufficiently evaluate the structure of the

150 acquired cystic lung disease. However, CT could be utilised to further evaluate the lesions at a

151 higher resolution, which may provide important anatomic information necessary in choosing a

152 therapeutic strategy.

153 In conclusion, most case reports of acquired cystic lung disease underlying BPD are

154 PIE, and bulla is rarely involved in acquired cystic lung disease. To decide the therapeutic

155 strategy, CT is useful when chest radiograph is equivocal on the evaluation of acquired cystic

156 lung disease.

157

158 List of abbreviations

159 BPD, bronchopulmonary dysplasia; PIE, pulmonary interstitial emphysema; CT, computed

160 tomography

161

162 Declarations

163 Ethics approval and consent to participate: Informed consent was obtained from patient.

164 Consent for publication:We obtained the consent for publication from the parent.

165 Availability of data and materials: Not applicable

166 Competing interests: The authors declare no conflict of interest.

167 Funding: Not applicable

168 Authors' contributions: TS,YH,KK and NO interpreted the patient data regarding the lung

169 disease. TO performed the histological examination of the bulla, and TS,KK were major

170 contributor in writing the manuscript. All authors read and approved the final manuscript. 
171 Acknowledgments: We would like to thank the members of the Department of Neonatology,

172 Paediatrics, Paediatric Surgery, Pathology, and Radiology.

175 References

176 1. Azizkhan RG, Grimmer DL, Askin FB, Lacey SR, Merten DF, Wood RE. Acquired lobar 177 emphysema (overinflation): Clinical and pathological evaluation of infants requiring 178 lobectomy. J Pediatr Surg. 1992;27:1145-51.

179 2. Srinivasan R, Ali H, Harigopal S. Persistent pulmonary interstitial emphysema presenting 180 as solitary lung cyst in a preterm infant. BMJ Case Rep. 2012. PMCID: PMC4543459.

181 3. Matta R, Matta J, Hage P, Nassif Y, Mansour N, Diab N. Diffuse persistent interstitial 182 pulmonary emphysema treated by lobectomy. Ann Thorac Surg. 2011;92:73-5.

183 4. Rao J, Hochman MI, Miller GG. Localized persistent pulmonary interstitial emphysema. J 184 Pediatr Surg. 2006;41:1191-3.

185 5. Firinci F, Duman N, Ates O, Ozer EA, Kumral A, Erdemir A, et al. Case of acquired lobar 186 emphysema mimicking pneumothorax in a neonate. East Mediterr Health J. 2013;19:960$187 \quad 1$.

188 6. Rocha G, Flôr-de-Lima F, Azevedo I, Guimarães H. Severe bronchopulmonary dysplasia 189 with large pneumatoceles in an extreme preterm newborn. Rev Port Pneumol (2006). $190 \quad 2017 ; 23: 170-2$.

191 7. Hasleton PS. Spencer's pathology of the lung. 5th ed. Cambridge: Cambridge University 192 Press; 1996. p. 613-4.

193 8. Krishnamohan P, Shen KR, Wigle DA, Allen MS, Nichols FC 3rd, Cassivi SD, et al. 194 Bullectomy for symptomatic or complicated giant lung bullae._Ann Thorac Surg. $195 \quad 2014 ; 97: 425-31$. 
196 9. Aramini B, Ruggiero C, Stefani A, Morandi U. Giant bulla or pneumothorax: How to 197 distinguish._ Int J Surg Case Rep. 2019;62:21-3.

198 10. Agrons GA, Courtney SE, Stocker JT, Markowitz RI. From the archives of the AFIP: Lung 199 disease in premature neonates: radiologic-pathologic correlation. RadioGraphics. $200 \quad 2005 ; 25: 1047-73$.

201 11. Ghattas C, Barreiro TJ, Gemmel DJ. Giant bullae emphysema. Lung. 2013;191:573-4.

202

203 Figure legends

204 Figure 1

205 Chest computed tomography taken at 5 months of age demonstrated a large $30 \times 25 \mathrm{~mm}$

206 monocystic space with a clear rim (asterisk) and multiple cystic air spaces with a line-and-dot

207 pattern (white arrowheads) in the upper right lobe (a, b).

208

209 Figure 2

210 A chest radiograph revealed a giant cyst with a diameter of $80 \times 60 \mathrm{~mm}$, causing left

211 mediastinal left shift and left lung atelectasis.

212

213 Figure 3

214 Histopathologic analysis of the excised bulla wall (black arrows), visceral pleura (black

215 arrowheads), and lung parenchyma. The wall of bulla was located below the visceral pleura

216 adjacent to the lung parenchyma and was formed by fibrous tissue (Elastic Van Gieson

217 staining, $\times 200)$.

218 


\section{Figures}
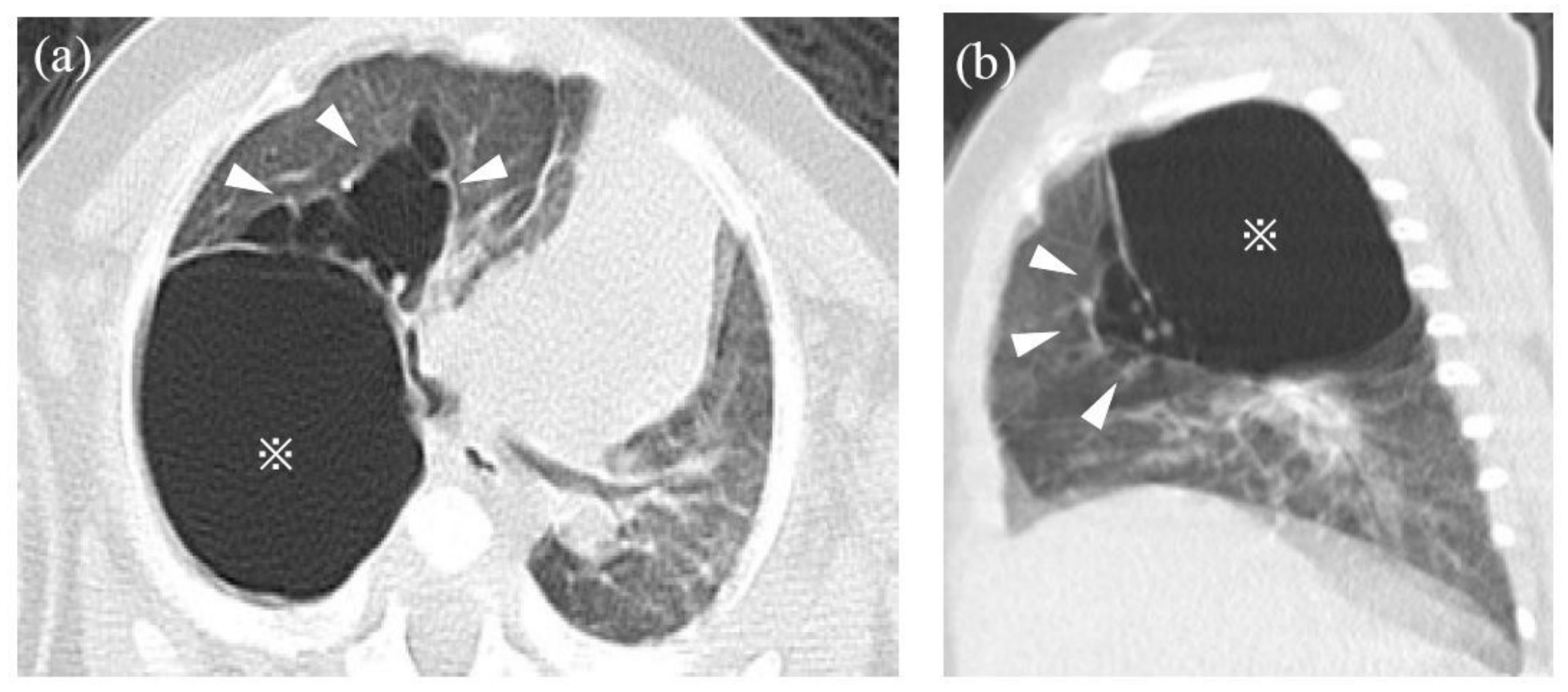

\section{Figure 1}

Chest computed tomography taken at 5 months of age demonstrated a large $30 \times 25 \mathrm{~mm}$ monocystic space with a clear rim (asterisk) and multiple cystic air spaces with a line-and-dot pattern (white arrowheads) in the upper right lobe $(a, b)$. 


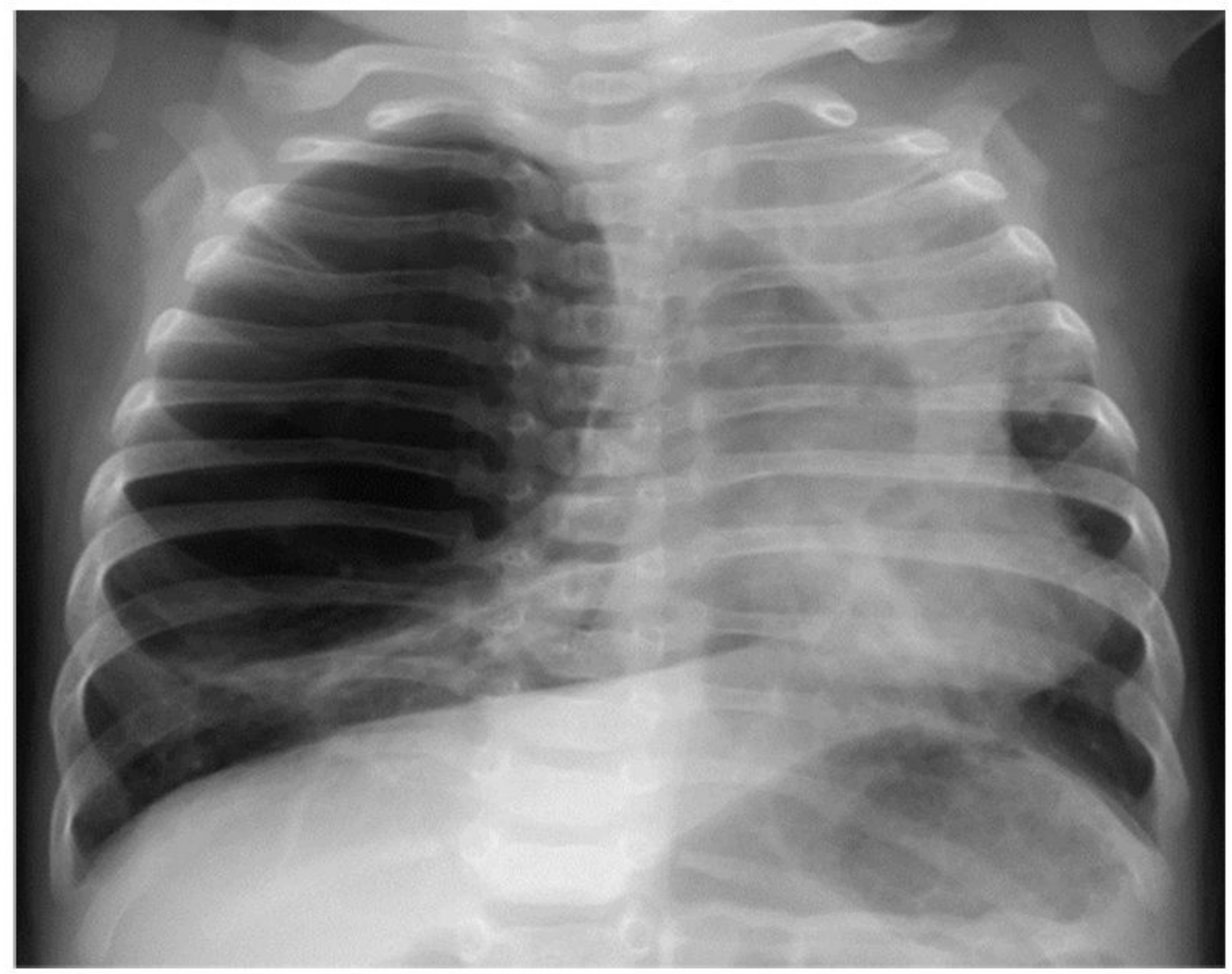

Figure 2

A chest radiograph revealed a giant cyst with a diameter of $80 \times 60 \mathrm{~mm}$, causing left mediastinal left shift and left lung atelectasis. 


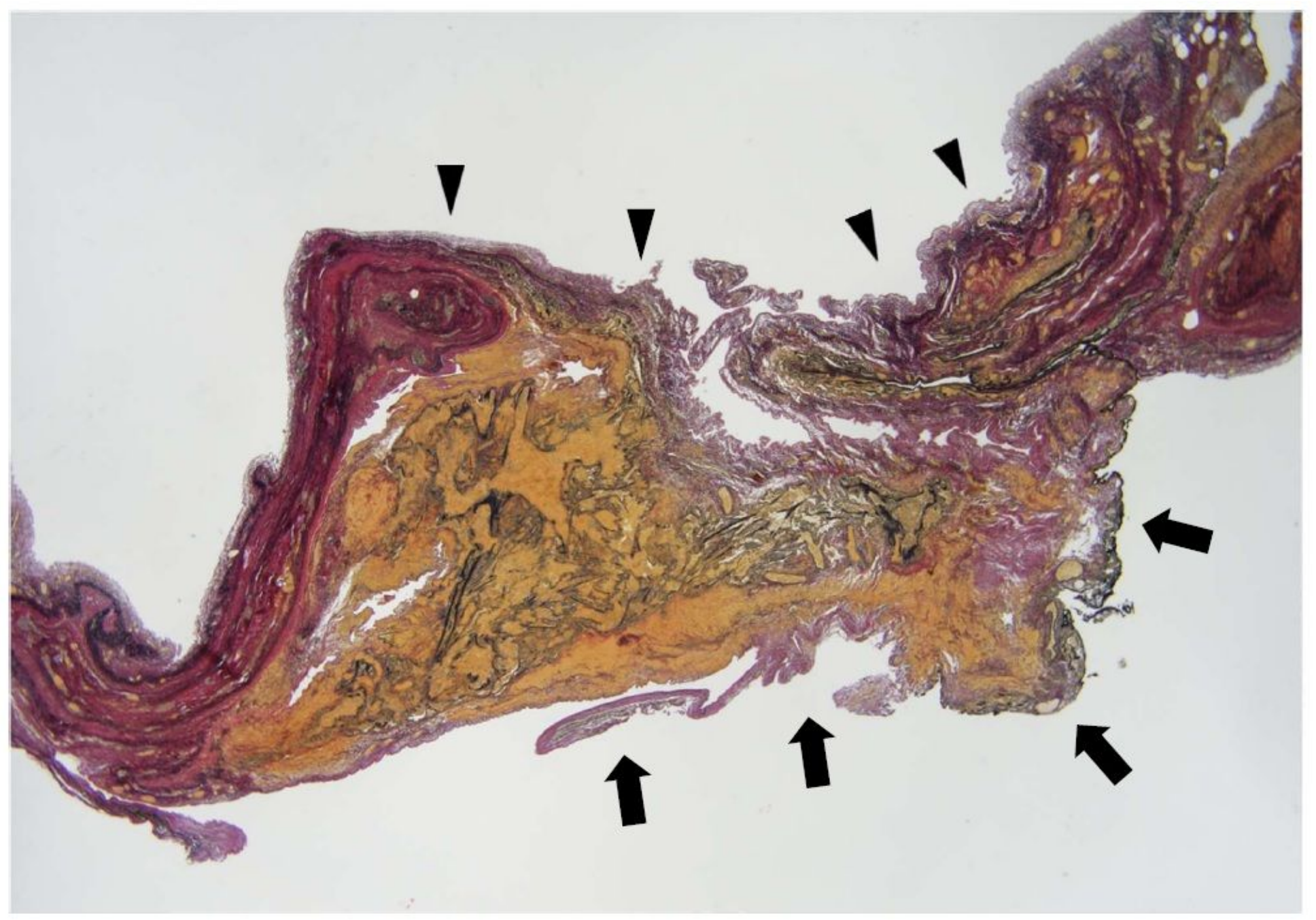

\section{Figure 3}

Histopathologic analysis of the excised bulla wall (black arrows), visceral pleura (black arrowheads), and lung parenchyma. The wall of bulla was located below the visceral pleura adjacent to the lung parenchyma and was formed by fibrous tissue (Elastic Van Gieson staining, $\times 200$ ). 